\title{
DIGITALCOMMONS
}

@WAYNESTATE-

Wayne State University

$1-1-2015$

\section{Design Ideas, Reflection, and Professional Identity: How Graduate Students Explore the Idea Generation Process}

\author{
Alisa Hutchinson \\ Wayne State University, ahutchina@gmail.com \\ Monica W. Tracey \\ Wayne State University, Monicatracey@wayne.edu
}

\section{Recommended Citation}

Hutchinson, A., \& Tracey, M. W. (2015). Design ideas, reflection, and professional identity: how graduate students explore the idea generation process. Instructional Science, 43(5), 527-544.

Available at: http://digitalcommons.wayne.edu/coe_aos/16 
Design ideas, reflection, and professional identity:

How graduate students explore the idea generation process

\author{
Alisa Hutchinson \\ Wayne State University \\ Monica W. Tracey \\ Wayne State University
}




\begin{abstract}
Within design thinking, designers are responsible for generating, testing, and refining design ideas as a means to refine the design problem and arrive at an effective solution. Thus, understanding one's individual idea generation experiences and processes can be seen as a component of professional identity for designers, which involves the integration of knowledge, action, and being in support of the professional self. Using written journal responses from graduate students in an introductory course in instructional design, this study explored how students used reflection to reconstruct experiences relating to the emergence of design ideas. Findings indicate that students were able to use reflection in support of professional identity development concerning design ideas, although attention to emotional aspects was lacking and many struggled to move from descriptive writing to meaning-making. Implications for professional identity development for designers and for future research are discussed.
\end{abstract}

Keywords: instructional design, design thinking, reflection, professional identity, idea generation, sources of design inspiration, graduate education 


\section{Background}

Design thinking positions the designer as the central driving force of the design process, responsible for engaging in an ongoing reflective conversation with an ill-structured problem in order to move through the design space toward a solution (Boling, 2008; Cross, 2011; Lawson, 2006; \& Nelson \& Stolterman, 2012). To maintain momentum in the design space, designers must conceptualize, select, test, and revise their ideas for tentative solutions in order to better understand the problem, which in turn supports the realization of an innovative solution. Thus, in order to develop a rich and dynamic sense of professional identity, or what Dall'Alba (2009) described as the integration of knowing, acting, and being in service of the professional self, designers would be well served to understand and continually reflect on how their own idea generation processes unfold and influence design actions and outcomes.

Despite their importance in maintaining design progress, much remains to be discovered about how ideas are generated in the design space, including what activities or stimuli might trigger the awareness or formation of a design idea (Chandrasekera, Vo, \& D'Souza, 2013; Gonçalves, Cardoso \& Badke-Schaub, 2014). The importance of sudden inspiration to design is a fixture of design thinking (Cross, 2007) and can be characterized as the point of emergence of a new idea that significantly accelerates or alters the direction of the design (Chandrasekera, Vo, \& D'Souza, 2013). It is commonly thought that inspiration strikes when intense work sessions are alternated with periods of mental relaxation away from the design problem (Cross, 2007) or, similarly, after a period of incubation or shift in context, often after a period of feeling blocked or fixated on an unworkable idea (Smith, 2003). Other studies have examined how stimuli may trigger the emergence of design ideas or influence the content of those ideas; these include textbased stimuli, visual stimuli, subliminal stimuli, verbal or conversational stimuli, and other idea 
generation activities (Cai, Do, and Zimring, 2009; Chandrasekera et al, 2012; Goldschmidt \& Sever, 2010; Gonçalves, Cardoso, \& Badke-Schaub, 2014; Perttula \& Sipilä; 2007; Salter \& Gann, 2002). Still others have identified the end users as the primary trigger for design ideation, with designers attempting to reduce the design problem complexity by empathizing with learners and better anticipating the learning experience (Rapanta \& Cantoni, 2014). However, the research literature on design ideas is still emerging (particularly within instructional design), with more attention needed to the methods that designers use to generate and evaluate ideas, as well as insight into how they characterize and understand their individual idea generation processes.

Likewise, professional identity development in design (for instruction or otherwise) has not been adequately explored in the academic literature at this juncture. The establishment and maintenance of professional identity involves the process of becoming, which addresses not only what we know and do but also who we are; despite this, most education programs stop short by focusing on the development of specific knowledge and skills without addressing the concurrent transformation the self (Dall'Alba, 2009). This transformation is typically achieved via external, socially situated representations of the self through dynamic narratives that are integral to developing professional identity and ways of being (Luehmann, 2007). For designers, professional identity includes the understanding of the self in the design sphere and what is expected of them within that space. In order to fully conceive of themselves as professionals, designers must know not only what design is and what designers do, but also who they are as a designer - how their personal traits, habits, talents, and limitations affect (and are affected by) their professional work. 
As such, this study is concerned with how students in a graduate-level instructional design class document and understand the sources of their ideas through reflective writing as an initial step in the process of recognizing themselves as professional designers. Drawing on a larger, design-based study on professional identity development and reflection in instructional design, (Author 2013; Author, 2014), this analysis is focused on design ideas as a specific component of designer identity development. As the data are retrospective accounts of subjective experiences, the results will be focused on understanding how participants think about the idea generation process, rather than providing an objective survey of what happens in the design space. Our research questions include:

1. How do participants engage in reflection when they respond to prompts about the idea generation processes?

2. What types of stimuli (external objects or influences, internal references, or personal behaviors) did participants identify as being associated with the emergence of ideas?

\section{Methodology}

As stated, this data is drawn from a larger, design-based study on professional identity development and reflection in instructional design (Author, 2013; Author, 2014). This analysis is focused on design ideas as a specific component of designer identity development. Data sources included online reflection journals which were analyzed using two rubrics, the REFLECT rubric (Wald, Borkan, Taylor, Anthony, \& Reis, 2012), a model for assessing reflective writing, and the Stimuli Promoting Design Ideas rubric, developed specifically for this study.

\section{Participants and context}

Participants included 69 master's and doctoral students enrolled in an introductory instructional design course held online over four semesters by a large research university in an 
urban area of the Midwestern United States. Most participants were enrolled in an instructional technology degree program, although students in other fields also take the course, as it is a requirement for an online teaching certificate offered by the graduate school. Given this mix, participants varied in age, academic background, and professional work experience. The subject pool also included several international students with varying English language skills, although all had the required proficiency necessary to be successful in the course.

The instructional technology department at this university operates within the design thinking tradition; as a first-semester requirement, this class is structured to support students in locating instructional design (ID) within the larger design community of practice. During the first half of the course, students learn general principles of design using Cross (2007) as their primary text; in the second half, ID principles are integrated into the larger design context established in prior weeks. In tandem with this framework, assignments are constructivist in nature and focused on solving ill-structured problems via personal reflection journals, case studies, prototyping, and peer group work.

\section{Data sources}

As mentioned, students kept a personal reflection journal as an ongoing assignment throughout the semester, with writing assignments during eight of the class's fourteen weeks. Students were given specific prompts as scaffolding for these assignments, covering assigned reading and case studies as well as prompts designed to support students in exploring concepts, beliefs, and experiences relevant to their emerging professional identities as instructional designers (see Author, 2013, and Author, 2014, for more detail). Journals were kept online in Google documents, with reading and editing rights granted to the course instructor in order to 
provide formative feedback and summative grades. This study focused on two prompts relevant to the purpose of the research as illustrated in Table 1.

[Insert Table 1 about here]

Prompt 3.3, which dealt with sudden inspiration, was the third prompt included in the third week of the course, and asked students to describe a personal experience that was akin to the sudden inspiration process described in Cross (2007) and lecture slides from that week. It is important to note that participants were not required to write about a design-related inspiration, but rather to describe an experience from any area of their lives. Because this was early in the semester, the instructional rationale was to provide students with the opportunity to make broad connections between their personal histories and experiences they would encounter in design, as a tentative foundation for identity building. As Table 1 demonstrates, the prompt was reworded during Semester 3, to break it up into two distinct questions, which were coded separately according to the data coding process described below. This prompt was then was further edited in Semester 4 to focus solely on the sudden inspiration question.

Prompt 6.6, relating to idea sources, was the sixth prompt included during the sixth week of the course. Participants also completed a case study reflection assignment that week, which asked them to identify key factors, challenges, and supports in an instructional design problem as well as describe the relationships among them via a concept map. After completing those tasks, students were asked to document their design ideas for this case study as well as how they came up with the ideas. This prompt was not included during Semester 4, so only data from Semesters 1-3 is included. 


\section{Data collection}

At the end of the semester, the instructor saved participating students' journals as Word documents, removed all identifying information, and forwarded them to the research team who assigned each subject a number. Responses to relevant prompts were extracted from individual journals and aggregated by prompt and semester into separate files (i.e., all responses to Prompt 3.3 from Semester 2 were captured in the same file, with each response identified only by subject number). No master list was kept associating names with subject numbers, so the final response sets were anonymous.

\section{Assessment procedures}

All responses were assessed using the REFLECT rubric (Wald, Borkan, Taylor, Anthony, \& Reis, 2012), which provides criteria for four levels of reflection across six criteria; see Table 2 for the full rubric. The four categories of reflection include habitual action (simple fact reporting and/or omission of major response elements); thoughtful action (more elaborate narrative but still remaining at the descriptive level without reflection); reflection (some effort to engage in questioning, meaning-making, analysis or some other aspect of reflection, beyond fact reporting); and critical reflection (a comprehensive critical response that considers multiple interpretations or viewpoints). The six criteria include writing spectrum (overall quality of the response); presence (sense of the author in the response); description of conflict (quality of the description of the precipitating event or conflict); emotion (exploration of emotional aspects); analysis (level of meaning-making in the response); and attention to assignment (how well the response addresses the prompt or task). Table 2 provides specific criteria for each reflection level in all six categories. 
For responses to the idea sources prompt, a second rubric, the Stimuli Promoting Design Ideas rubric was developed by the lead data coder, who read through the full response set and developed a list of nine categories directly drawn from participant responses, along with the options to code for "did not indicate," "could not pinpoint" (for cases where participants specifically stated that they did not know where their ideas came from), and "other," for a total of 12 categories. As listed in Table 4, the categories included: emerged while considering factors, causes, and/or obstacles specific to the problem; emerged while considering prior experiences/knowledge/examples; emerged while developing concept map/making notes/other artifacts or sketching; emerged while considering target audience/end users; emerged while trying to better define problem(s); emerged while considering the whole system/environment; emerged while considering first principles; emerged after a period of rest; and emerged while prioritizing goals. Coders were instructed to select as many options as needed to capture all idea sources in a given response.

For each coding decision, every response was evaluated by at least two coders. If those coders disagreed, the decision was sent to a third coder; in rare cases where the first three coders disagreed, the decision was sent to the primary researchers for final adjudication. The coding team included seven coders who were graduate students in instructional technology or educational psychology, although experience with data coding and knowledge of design thinking varied among them, as well as a lead coder who was a doctoral student with significant experience coding reflection data in relation to designer identity concepts.

\section{Results}

\section{Student reflection on idea generation processes}


Our first research question was designed to uncover how students use reflection to explore their idea generation processes, using the reflection levels and criteria included in the REFLECT rubric. A total of 79 responses were included for Prompt 3.3, which asked participants to describe an experience with sudden inspiration; this number is slightly higher than our total $\mathrm{N}$ of 69 because the prompt was split into two questions for Semester 3 (see Table 1 for more detail). A total of 46 responses were included for Prompt 6.6, which asked students to document their design ideas and how they came up with them; because this prompt was not included in Semester 4, the number of responses was lower than our total $\mathrm{N}$ of 69 . Thus, there were a total of 125 responses, each of which were evaluated on the six criteria of the REFLECT rubric, for a total of 750 coding decisions. Table 3 provides full detail on reflection level assessments for each criterion by prompt and semester.

[insert Table 3 about here]

\section{Aggregated reflection levels}

Aggregated reflection levels for all criteria across both prompts demonstrated that $43 \%$ of responses were coded as reflection, followed by $38 \%$ as thoughtful action, $17 \%$ as habitual action, and $2 \%$ as critical reflection, as shown in Figure 1.

[insert Figure 1 about here]

\section{Reflection levels by REFLECT criteria}

Figure 2 plots reflection level percentages for each criterion in the REFLECT rubric (aggregated from both prompts).

[insert Figure 2 about here]

When considering reflection levels by REFLECT criteria, the Presence criterion (which indicates the level of authorial presence in the response) demonstrated the highest levels of reflection as 
well as the lowest levels of thoughtful and habitual action. The Emotion criterion (which indicated the inclusion of emotional factors in the response) included the lowest level of reflection and the highest level of habitual action but interestingly it also had the second highest level of critical reflection.

After Presence (mentioned above), the Analysis criterion had the highest levels of reflection followed by Conflict, Writing Spectrum, and Attention to Assignment. For thoughtful action, the highest level was seen in Attention to Assignment, followed by Conflict, Analysis, Writing Spectrum, Presence, and finally Emotion. After Emotion (discussed above), the highest levels of habitual action were seen in Writing Spectrum, Attention to Assignment, and Analysis, followed equally by Presence and Conflict. Finally, although 5\% or less in each category, critical reflection was coded at the highest rate for Writing Spectrum, followed by Emotion, Analysis, and Conflict; the Presence and Attention to Assignment criteria did not include an option for critical reflection.

\section{Reflection levels by prompt}

As indicated in Figure 3, when comparing aggregated reflection levels between the two prompts, Prompt 3.3 demonstrates higher levels of reflection compared to Prompt 6.6, although levels of critical reflection are the same. Habitual action was coded more frequently for Prompt 6.6 versus Prompt 3.3, and thoughtful action was coded more frequently for Prompt 3.3 than Prompt 6.6. See Figure 4 for more detail.

[insert Figure 3 about here]

\section{REFLECT criteria by prompt}

Figure 4 illustrates the changes within REFLECT criteria from Prompt 3.3 to Prompt 6.6. Three of the dimensions held relatively steady across prompts: emotion, analysis and writing 
spectrum. The remaining three dimensions saw a shift toward less reflection and more descriptive action with presence demonstrating the most significant shift in quality. See Figure 4 for more detail.

[insert Figure 4 about here]

\section{Stimuli promoting design ideas and inspiration}

In addition to the REFLECT rubric, responses to Prompt 6.6 were coded using the Stimuli Promoting Design Ideas rubric, to identify stimuli that were associated with the emergence of design ideas and inspiration. Twenty-six percent of responses either didn't indicate any associated stimuli or specifically stated that idea sources were unknown, while $25 \%$ indicated that ideas emerged while considering factors, causes, and/or obstacles specific to the problem and $21 \%$ indicated that ideas emerged while considering prior experiences, knowledge, or examples. The remaining $28 \%$ were distributed among seven other categories included in the coding rubric; see Table 4 for more details.

[insert Table 4 about here]

\section{Coder agreement rates for both rubrics}

Because these rubrics address distinct aspects of participant responses and because they were developed separately, coder agreement rates were independently calculated for both. Of the 750 coding decisions using the REFLECT rubric, 94\% achieved agreement from two out of three reviewers, with $6 \%$ sent to the principle researchers for final adjudication. From the 42 responses coded for design idea sources using the Stimuli Promoting Design Ideas rubric, 78\% achieved agreement between two out of three reviewers, with the remaining $22 \%$ referred to the primary researchers for final adjudication.

\section{Discussion and implications}


It is clear from the results that many participants were able to engage in reflection when responding to Prompt 3.3 (47\% average across all dimensions for reflection and $2 \%$ for critical reflection), which dealt with sudden inspiration. Participant reflection was somewhat diminished when addressing idea sources in response to Prompt $6.6(36 \%$ average across all dimensions for reflection and $2 \%$ for critical reflection). Relative to both prompts, students struggled to incorporate reflection on emotional aspects in their written narratives, with only $15 \%$ achieving reflection or critical reflection for Prompt 3.3 and only 9\% for Prompt 6.6 for the Emotion criteria. The following are insights and implications in light of these findings.

\section{General patterns in student reflection}

The aggregated results show that reflection was the most common level achieved by participants, followed by thoughtful action, habitual action, and critical reflection. When reflection and critical reflection are considered against thoughtful action and habitual action, however, a small majority of the total coding decisions (55\%) fall under the latter two, which suggests that most responses were pre-reflective in relation to the criteria of the REFLECT rubric. Pre-reflection (Davis, 2006; Author, 2013) is a useful way of considering student responses by framing habitual and thoughtful action as containing the seeds of reflection, which could be developed into reflection given proper scaffolding and feedback. In fact, one of the purposes of the REFLECT rubric was to provide a framework for formative feedback that could support students in developing their reflective capacity (Wald et al, 2012). It is also important to point out that professional identity construction is not a stable, straightforward process (Dall'Alba, 2009; Luehmann, 2007), and thus reflective writing done in service of this task will likely reflect this instability. Our preliminary research on reflection and identity development supports this, as results suggested that reflective abilities varied within individual participants 
across the semester, particularly in participants who fell in the lower tiers of reflective ability (Author, 2014).

While aggregated results are useful for seeing general patterns in the data, they can also mask interesting differences that occur within the details. Fruitful implications emerge when (1) considering differences in reflection by comparing REFLECT criteria against each other and (2) considering differences by comparing reflection levels elicited by the two prompts included in the study.

\section{Variation in reflection levels by REFLECT criteria}

Presence, or the sense of the author in the response, was the criterion most commonly coded as reflective, with $67 \%$ of responses coded as reflection vs. $52 \%$ for Analysis coded as reflection or critical reflection. However, it could be argued that this result seems somewhat low, as both prompts asked participants to describe a personal experience which would seem to demand the author's presence in the response yet only two-thirds of responses were considered to contain a "sense of the writer being largely or fully present" (see Table 2). As discussed previously, professional identity involves the integration of knowledge, action, and being (Dall'Alba, 2009) which implies that finding and using one's personal voice as a conduit for external representations of the self is fundamental to identity development work. Our results suggest emerging instructional designers may need support in developing their personal voice as well as maintaining its presence in reflective activities, through the affordance of multiple opportunities to reflect on professional knowledge, experiences, and actions as well as feedback to improve the incorporation of personal presence in such reflection.

Turning now to the criterion coded as least reflective, the Emotion criterion saw only $14 \%$ of responses reaching reflection or critical reflection, with $54 \%$ categorized as habitual 
action (as a note of comparison, the next highest percentage for habitual action was Writing Spectrum at only 14\%). Interestingly, Emotion also had the second-highest percentage of responses coded as critical reflection at $4 \%$. These results strongly indicate that participants did not routinely attend to affective issues that may have been relevant to their responses, although when they did move into reflection, they were able to achieve emotional insight almost $30 \%$ of the time. While neither prompt specifically asked students to reflect on their emotions, both referenced situations that naturally contain affective elements. For example, Prompt 3.3 asks students about mental relaxation (which implies the absence or relief of anxiety, an emotion) as well as a moment of sudden inspiration, which often includes an element of thrill or excitement. While the situation embedded in Prompt 6.6 is not as directly connected to the emotions, it would not be unexpected for participants to have encountered frustration, anxiety, relief, or even elation as they came up with their design ideas.

Initial empirical investigations on emotion and designers support the idea that designer affect may help shape design processes, decisions, and outcomes (Davis, 2009; DeDru, Baas \& Nijstad, 2008; Yang \& Hung, 2014). As such, the ability to incorporate emotional responses into design-driven reflection is important for two reasons: (1) professional identity development, regardless of the field, involves understanding the self (including the emotional self) in the context of professional responsibilities and (2) affective states can influence design thinking and creativity, which implies a potential effect on design outcomes (Dong, Kleinsmann, \& Valkenburg, 2009; Solovyova, 2003). Developing emotional self-awareness may be also be crucial in design because empathy, which involves the development of emotional connections and understanding, serves as an important bridge between the designer and the user (Kouprie \& Visser, 2009). Although individual designers vary in their ability to engage in empathy, research 
indicates that empathizing with end users during the design permits instructional designers to better anticipate the user learning experience, consequently strengthening the final design (Rapanta \& Cantoni, 2014). Thus, our results imply that students need significant support via scaffolding and feedback in order to recognize, attend to, and gain insight into the emotional components that influence design thinking, actions, and outcomes.

\section{Variation in reflection levels by prompt}

A comparison of reflection levels by prompt suggests that participants found it easier to achieve reflection in response to Prompt 3.3 (sudden inspiration) than Prompt 6.6 (ideation stimuli). Just under half of the coding decisions for Prompt 3.3 were categorized as either reflection or critical reflection, while only $38 \%$ of Prompt 6.6 decisions were coded at these levels. Furthermore, just over a quarter of Prompt 6.6 decisions were coded as habitual action while only $11 \%$ of Prompt 3.3 decisions were.

A few plausible explanations seem appropriate to consider. First, it can be difficult for designers (even those with significant experience) to identify and cogently discuss how their design ideas emerged (Cross, 2011); this concept is discussed in more detail below, in relation to participants' identification of idea sources. Second, the text of Prompt 3.3 allowed students to choose an experience from any point in their history and was not limited to the design space. On the other hand, Prompt 6.6 was asking participants to reflect on a complex activity that had just occurred and that was unfamiliar to many of them. The lack of chronological distance from the experience may have meant that some students were still absorbing the cognitive and emotional consequences of the activity, influencing their ability to generate meaningful reflection and map the connection between personal experience and larger design concepts. Third, it is possible that the complexity of Prompt 6.6 itself, along with the case study activity that preceded and served 
as its subject, increased cognitive load to the point where resources available for reflection were diminished. Finally, in these activities guiding the development of professional identity, we provide opportunities for students to transform themselves by placing them in a reflective place where they may be emotionally challenged (Thomson, 2004). Indications are that this process, begun through the reflection prompts, needs to be expanded to allow the student more time in this reflective place; this can be supported by incorporating multiple opportunities for reflection throughout the curriculum.

\section{Understanding stimuli promoting design ideas and inspirations}

Responses that either didn't indicate the source of design ideas or specifically stated that idea sources were unknown represented the largest group $(\mathrm{N}=16)$, although they were closely followed by responses that referred to consideration of some aspect of the design problem as the source of design ideas $(\mathrm{N}=15)$ and those that referred to prior experiences, knowledge, or examples as the source $(\mathrm{N}=13)$. As mentioned previously, it can be difficult for designers, experienced and novice alike, to articulate the complex and often unconscious processes that lead to the emergence of design ideas and these results are in line with this. As a caveat, conflating graduate student status with novice status is not always accurate, but in this case it is likely that even experienced graduate students would also struggle with this topic. While many responses attributed the emergence of ideas to the consideration of the constraints inherent in the design process, this doesn't necessarily tell us much about what the participants were doing at the time, or how they were considering the problem. Were they brainstorming, sketching, discussing with a peer? Understanding the connection between the problem and tentative ideas for the solution is a critical first step in gaining design momentum, but it may also be important to encourage consideration of the specific activities that leverage this connection to generate ideas. Also 
telling is the role of personal experience in generating ideas, which underscores the importance of professional identity as an avenue for a dynamic, recursive understanding of the translational relationship between the self (including personal history) and the design sphere, as well as the influence of personal experiences and beliefs on design outcomes.

It is also important to note that this study was the pilot for the second rubric, the Stimuli Promoting Design Ideas rubric, used to identify design idea sources in subject responses. The inter-rater agreement rate of $78 \%$ for this rubric (for two out of three coders) indicates that further development of the rubric is necessary to improve its validity and reliability. At the same time, these preliminary results are encouraging and support refinement of the instrument.

\section{Limitations of the Study}

Our study draws on retrospective reflective writing by graduate student participants, thus it represents subjective interpretations of events and ideas rather than objective accounts of actions taken in the design sphere. While subjective accounts provide valuable information about how individuals understand and narrate important issues to themselves and others, it is important to remember that they also carry biases and do not necessarily tell us what participants actually do, but rather what they think they do (or would perhaps like to do, on a subconscious level). Furthermore, it is important to remember that our subject pool was relatively small ( $\mathrm{N}=79$ for Prompt 3.3, and $\mathrm{N}=46$ for Prompt 6.6 ) and limited to students in an instructional design course, which dictates caution in generalizing the results, both within instructional design as well as to other fields.

\section{Implications for professional identity development}

The results from this study lend support to the importance of including professional identity development as a component of graduate-level design curricula as well as the role of 
reflective writing as a tool for identity development. While we are responsible for teaching a student what he should know and do, we are equally responsible to teach a student how to become a professional. Heidegger (1962) emphasizes that the central purpose of education is the transformation of the self and states that real education transforms the professional identity of a student. Reflection journals in this graduate level design course are the mechanism for students to challenge their assumptions, articulate their actions and reflect on design ideas, in an effort to develop designer identity. Therefore while reflection levels may be more representative of an individual student's experiences and/or writing skills, the value comes from giving all students the opportunity to practice and build confidence in their ability to articulate experiences with and beliefs about design processes. Within the design thinking tradition, it is the designer who generates, selects, tests, and refines ideas in order to better understand the problem and develop solutions. Thus, it is important for designers to understand how they influence this process via their personal experiences, beliefs, and actions, particularly as they relate to the affective aspects of the design process (which participants in our study routinely failed to incorporate in their responses, even when it would have been logical and natural to do so). The lack of attention to emotion in participants' responses also highlighted the need to further study how emotional selfawareness influences designer identity development, design actions, and design outcomes.

Instructional design provides a fertile test ground for exploring professional development for designers, because the field has only recently made a significant shift to align with design thinking and move away from earlier, process-driven models of the field (Author, 2013). As such, it is an ideal time to also begin exploring professional identity development in tandem with the evolution of the discipline's self-definition. Within the general design literature, identity development has not been well researched or explored to date, and thus the lessons learned as 
our discipline incorporates issues of professional identity in graduate education may be important touch points for other fields. All design disciplines could benefit from more research on how to foster dynamic identity development (and why it matters) in fields beyond instructional design, both in emerging and established design professionals. The knowledge generated will be crucial to developing a broader and deeper understanding of what it means to be a designer, both in a global sense and in how identity might vary based on design discipline.

\section{Implications for development of student designers}

It is also important to consider ways that instructors can elevate the quality of student responses via scaffolding (such as prompts) and formative feedback. As mentioned, the REFLECT rubric was intended as a tool to develop effective feedback practices by providing a framework for understanding important components of reflection as well as graded levels of reflection. Rather than striving to have students all achieve a specific reflection level, the value is helping individual students understand where they are at and what they need to consider to deepen their understanding of who they are as instructional designers, thus assisting them in developing their professional identity. This means supporting students as they draw connections between personal experiences (both inside and outside of the design sphere) and the complex professional activities and responsibilities they will encounter in their design practice, while remaining sensitive and open to the multiple interpretations and viewpoints that are inherent in ill-structured design problems. In the case of design idea generation, providing students with a foundational understanding of how their design ideas might emerge, sows the seeds for an identity development process that will continue to unfold across their careers as designers.

The prompts included in this study were sequenced to provide students with scaffolding for development of reflection on design ideas, moving from a general example of inspiration to a 
specific accounting of idea emergence in relation to a design problem. However, reflection rates fell between prompts, indicating that students may need additional support in achieving reflection when challenged with increasingly complex situations and prompts. As this study is part of an ongoing, design-based project, changes have been made to the text of Prompt 6.6 for subsequent semesters, which will provide a basis for comparison and further insight into instructional conditions that foster reflection.

\section{Other research directions}

Finally, two other important implications emerged from our findings related to reflection on design ideas in particular. Difficulties in articulating the ideation process have been mentioned in the literature (Cross, 2011) and were also seen in these results relative to Prompt 6.6. More research is necessary to understand why this is the case, and furthermore, to uncover what value this understanding might contribute to design outcomes. Perhaps for some designers, preserving the mystery of the creative process is a protective act; on the other hand, providing designers with even a brief opportunity to explore their own process may generate lasting benefits, even if attempts to articulate this process are frustrating or abandoned. Emotional components of the idea generation process were largely neglected by our participants, yet affective processes are likely crucial to effective actions in the design sphere. More research is needed to understand how emotional self-awareness intersects with designer identity development as well as to refine our understanding of how designers' affective responses and actions influence idea generation and resulting design outcomes. 


\section{References}

Author, (2013).

Author, (2013).

Author, (2014).

Boling, E. (2008, October). Design is not systematic: Alternative perspectives on design Designer as human instrument. Panel session presented at the meeting of the Association for Educational Communications and Technology. Orlando, FL.

Chandrasekera, T., Vo, N., \& D'Souza, N. (2013). The effect of subliminal suggestions on Sudden Moments of Inspiration (SMI) in the design process. Design Studies, 34(2), 193215.

Cross, N. (2011). Design thinking. New York, NY: Berg.

Dall'Alba, G. (2009). Learning professional ways of being: Ambiguities of becoming. Educational Philosophy and Theory, 41(1), 34-45. doi10.1111/j.1469-5812.00475.x

Davis, E.A. (2006). Characterizing productive reflection among preservice elementary teachers: Seeing what matters. Teaching and Teacher Education, 22, 281-301. doi: 10.1016/j.tate.2005.11.005

Davis, M. A. (2009). Understanding the relationship between mood and creativity: A metaanalysis. Organizational Behavior and Human Decision Processes, 108, 25-38

De Dreu, C. K., Baas, M., \& Nijstad, B. A. (2008). Hedonic tone and activation level in the mood-creativity link: toward a dual pathway to creativity model. Journal of personality and social psychology, 94(5), 739. 
Dong, A., Kleinsmann, M., \& Valkenburg, R. (2009). Affect-in-cognition through the language of appraisals. Design Studies, 30(2), 138-153. doi: 10.1016/j.destud.2008.12.003

Goldschmidt, G., \& Sever, A. L. (2011). Inspiring design ideas with texts. Design Studies, 32(2), 139-155. doi:10.1016/j.destud.2010.09.006

Gonçalves, M., Cardoso, C., \& Badke-Schaub, P. (2014). What inspires designers? Preferences on inspirational approaches during idea generation. Design Studies, 35(1), 29-53.

Heidegger, M. (1962). Being and time. New York, NY: Harper \& Row, Publishers, Incorporated. Lawson, B. (2006). How designers think: the design process demystified ( $4^{\text {th }}$ ed.). Oxford, UK: Elsevier.

Luehmann, A.L. (2007). Identity development as a lens to science teacher preparation. Science Education, 91, 822-839. doi: 10.1002/sce20209

Mose Biskjaer, M., \& Halskov, K. (2014). Decisive constraints as a creative resource in interaction design. Digital Creativity, 25(1), 27-61. doi:10.1080/14626268.2013.855239

Nelson, H.G., \& Stolterman, E. (2012). The design way: Intentional change in an unpredictable world $\left(2^{\text {nd }}\right.$ ed. $)$ Cambridge, MA: MIT Press.

Perttula, M., \& Sipilä, P. (2007). The idea exposure paradigm in design idea generation. Journal of Engineering Design, 18(1), 93-102. doi: 10.1080/09544820600679679

Rapanta, C. \& Cantoni, L. (2014). Being in the users' shoes: Anticipating experience while designing online courses. British Journal of Educational Technology, 45(5), 765-777. doi:10.1111/bjet.12102

Salter, A., \& Gann, D. (2003). Sources of ideas for innovation in engineering design. Research Policy, 32(8), 1309-1324. 
Smith, S. M. (2003). The constraining effects of initial ideas. In Paulus, B., Nijstad, B.A., \& Nijstad, B.A. (Eds.), Group creativity: Innovation through collaboration. Cary, NC: Oxford University Press.

Solovyova, I. (2003). Conjecture and emotion: An investigation of the relationship between design thinking and emotional content. In Expertise in design: Design thinking research symposium (Vol. 6).

Thomson, I. (2004). Heidegger's perfectionist philosophy of education in being and time. Continental Philosophy Review, 37, 439-467. doi:10.10007/s11007-005-6886-8 Yang, J. S., \& Hung, H. V. (2014). Emotions as Constraining and Facilitating Factors for Creativity: Companionate Love and Anger. Creativity and Innovation Management. 